\title{
Structural and transcriptional evidence of mechanotransduction in the Drosophila suzukii ovipositor
}

\author{
Cristina Maria Crava ${ }^{\mathrm{a}, \mathrm{f}, *}$, Damiano Zanini ${ }^{\mathrm{b}, \mathrm{g}}$, Simone Amati ${ }^{\mathrm{a}}$, Giorgia Sollai ${ }^{\mathrm{c}}$, Roberto Crnjar $^{\mathrm{c}}$, \\ Marco Paoli $^{\mathrm{b}}$, Marco Valerio Rossi-Stacconi ${ }^{\mathrm{a}}$, Omar Rota-Stabelli ${ }^{\mathrm{a}}$, Gabriella Tait ${ }^{\mathrm{a}}$, \\ Albrecht Haase $^{\mathrm{b}}$, Roberto Romani ${ }^{\mathrm{d}, *, 1}$, Gianfranco Anfora ${ }^{\mathrm{a}, \mathrm{e}, 1}$ \\ ${ }^{a}$ Research and Innovation Centre, Fondazione Edmund Mach, San Michele all'Adige, Italy \\ ${ }^{\mathrm{b}}$ Center for Mind/Brain Sciences and Department of Physics, University of Trento, Rovereto, Italy \\ ${ }^{\mathrm{c}}$ Department of Biomedical Sciences, Section of Physiology, University of Cagliari, Italy \\ ${ }^{\mathrm{d}}$ Department of Agricultural, Food and Environmental Sciences, University of Perugia, Perugia, Italy \\ ${ }^{\mathrm{e}}$ Centre Agriculture, Food and Environment (C3A), University of Trento, San Michele all'Adige, Italy

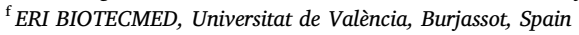 \\ ${ }^{\mathrm{g}}$ Neurobiology and Genetics, Biozentrum Universität Würzburg, Julius-Maximilians-University of Würzburg, Germany
}

\section{A R T I C L E I N F O}

\section{Keywords:}

spotted wing drosophila

mechanosensitive bristles

ultrastructure

comparative RNA-seq

\begin{abstract}
A B S T R A C T
Drosophila suzukii is an invasive pest that prefers to lay eggs in ripening fruits, whereas most closely related Drosophila species exclusively use rotten fruit as oviposition site. This behaviour is allowed by an enlarged and serrated ovipositor that can pierce intact fruit skin, and by multiple contact sensory systems (mechanosensation and taste) that detect the optimal egg-laying substrates. Here, we tested the hypothesis that bristles present in the $D$. suzukii ovipositor tip contribute to these sensory modalities. Analysis of the bristle ultrastructure revealed that four different types of cuticular elements (conical pegs type 1 and 2, chaetic and trichoid sensilla) are present on the tip of each ovipositor plate. All of them have a poreless shaft and are innervated at their base by a single neuron that ends in a distal tubular body, thus resembling mechanosensitive structures. Fluorescent labelling in $D$. suzukii and D. melanogaster revealed that pegs located on the ventral side of the ovipositor tip are innervated by a single neuron in both species. RNA-sequencing profiled gene expression, notably sensory receptor genes of the terminalia of D. suzukii and of three other Drosophila species with changes in their ovipositor structure (from serrated to blunt ovipositor: Drosophila subpulchrella, Drosophila biarmipes and D. melanogaster). Our results revealed few species-specific transcripts and an overlapping expression of candidate mechanosensitive genes as well as the presence of some chemoreceptor transcripts. These experimental evidences suggest a mechanosensitive function for the $D$. suzukii ovipositor, which might be crucial across Drosophila species independently from ovipositor shape.
\end{abstract}

\section{Introduction}

Drosophila suzukii (Matsumura) (Diptera: Drosophilidae), also called spotted wing drosophila, is an invasive South Eastern Asian fly species that was identified outside its native range in California in 2008, and in Spain and Italy in 2009 (Cini et al., 2012; Hauser, 2011). Since then, it has spread quickly across several countries in both continents, where it is now a major threat for soft fruit production (Asplen et al., 2015). Differently from the majority of drosophilids, which thrive and lay eggs on already damaged or rotting vegetal substrates, D. suzukii can pierce and lay eggs on healthy ripening fruits before they are harvested.
Wherever it is present, D. suzukii causes extensive agricultural damage, and this has boosted research on the ecology and chemosensory behaviour of this species with the aim to find innovative, effective, and ecofriendly methods to reduce its attacks (reviewed in (Cloonan et al., 2018)).

Several aspects of $D$. suzukii ecology and genetics have been analysed in a comparative framework across Drosophila species to identify key evolutionary innovations that allowed the transition from rotten to fresh fruit egg-laying behaviour (Atallah et al., 2014; Crava et al., 2016; Green et al., 2019; Hickner et al., 2016; Karageorgi et al., 2017; Muto et al., 2018; Ramasamy et al., 2016). The major morphological shift

\footnotetext{
* Corresponding authors.

E-mail addresses: m.cristina.crava@uv.es (C.M. Crava), roberto.romani@unipg.it (R. Romani).

${ }^{1}$ Authors share joint seniority.
} 


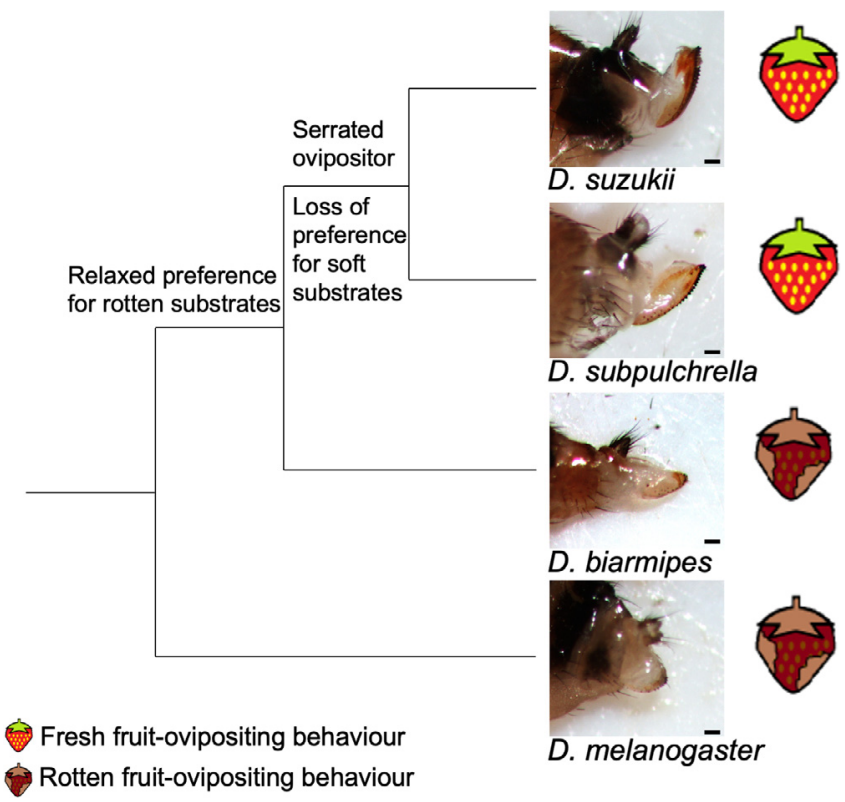

Fig. 1. Evolutionary affinities and ovipositor shapes of Drosophila suzukii and other Drosophila species used in the study. The currently most accepted scenario for fresh fruit-egg laying behaviour evolution (Karageorgi et al.,2017) is depicted. Phylogeny is based on Atallah et al. (2014). Scale bars: $100 \mu \mathrm{m}$.

from rotten fruit-ovipositing Drosophila species (like the insect model Drosophila melanogaster) to fresh fruit-ovipositing species (D. suzukii) is the presence of an enlarged and serrated ovipositor. Such structure is shared with the sister species Drosophila subpulchrella and allows the wounding of the intact skin of berries (Fig. 1) (Atallah et al., 2014). This feature is not present in another closely related Asiatic spotted wing Drosophila species, Drosophila biarmipes, whose ovipositor shows intermediate features between $D$. suzukii and D. melanogaster (Fig. 1) (Atallah et al., 2014). The ovipositor morphology correlates with the stiffness of the oviposition substrates, and serrated design facilitates egg-laying by attenuating the penetration force required to cut through the fruit skin; accordingly, $D$. melanogaster egg-laying is inhibited by stiff substrates, whereas $D$. suzukii has a broad tolerance, and D. biarmipes displays an intermediate behaviour (Fig. 1) (Karageorgi et al., 2017).

To detect substrate stiffness, insects rely on mechanosensitive sensilla. In Drosophila spp., these are scattered throughout the body and can be of different types such as bristles, hair plates, campaniform sensilla, and chordotonal organs (Karkali and Martin-Blanco, 2017). Bristles are the main touch receptors, while the other three organs are proprioceptors, i.e. mechanosensitive structures that monitor the positions, and relative movements of the fly's own body parts (Keil and Steinbrecht, 1984; Tuthill and Wilson, 2016). A feature common to all mechanosensitive sensilla is the presence of mechanosensitive neurons (MNs), which have an outer dendritic segment that ends in a distal tubular body in contact with the flexible base of the sensillum shaft (Walker et al., 2000). Pure mechanosensitive sensilla accommodate a single MN whereas in poly-innervated taste sensilla the $\mathrm{MN}$ is coupled with multiple gustatory neurons and four non-neural cells (trichogen cell, tormogen cell, thecogen cell and glial cell) (Falk et al., 1976; Stocker, 1994). While the tip of mono-innervated mechanosensitive sensilla is smooth, the one of taste sensilla has one or few pores that allow dendrites of gustatory neurons to get in contact with external fluid (Falk and Atidia, 1975). In D. melanogaster, poly-innervated taste sensilla have been found in the labial palps, the pharynx, the legs and the wings, but their presence on the external genitalia was only suggested (Stocker, 1994). In D. suzukii, a single study described the external morphology of the ovipositor tip (Atallah et al., 2014), but no information on the ultrastructrure of sensilla and pegs (which would provide clues for the physiological function of these structures) are available yet. Besides mechanosensation, also contact chemosensation is crucial for egg laying behaviour in insects, and in D. suzukii, tastants affect the selection of the optimal egg-laying site for (Karageorgi et al., 2017). Since the ovipositor of $D$. suzukii pierces the fruit skin and comes into contact with the fruit flesh, we hypothesized that this organ may carry taste sensilla, which contribute to the egg-laying decision. Alternatively, it is possible that D. suzukii ovipositor carries pure mechanosensitive sensilla, which may transfer information on the substrate stiffness and roughness, and on whether the ovipositor has penetrated it.

To select among these hypotheses, we analysed the ultrastructure of the pegs and sensilla present on $D$. suzukii ovipositor tip. We then used fluorescent antibody and GAL4 drivers to label neurons reaching these structures in both D. suzukii and D. melanogaster. Lastly, we performed RNA-seq experiment to understand if terminalia gene expression overlaps among Drosophila species characterised by gradual changes in their ovipositor structure (from blunt to serrated ovipositor) (Fig. 1). Our results reveal the presence of mechanosensitive sensilla in $D$. suzukii ovipositor and suggest that mechanotransduction in ovipositor is conserved among Drosophila species independently from ovipositor shape.

\section{Methods}

\subsection{Insects}

Insects used for transcriptomics, immunohistochemistry, and electron microscopy were taken from laboratory colonies maintained at the Fondazione Edmund Mach, S. Michele all'Adige (Italy). Drosophila suzukii and D. melanogaster strains were founded with individuals collected in 2010 in the Trento province (Italy) and periodically refreshed with insects caught from the same field sites. Drosophila biarmipes (genotype Dbii \wild-type, stock \# 14023-0361.09) and D. subpulchrella (Dspc \wild-type, stock \# 14023-0401.00) strains were obtained from the Drosophila Species Stock Center (San Diego, CA, US) in 2011. The four Drosophila species were reared on a standard diet (https:// stockcenter.ucsd.edu/info/food_cornmeal.php), maintained at $23-25{ }^{\circ} \mathrm{C}, 65 \pm 5 \%$ relative humidity, and under a 16:8 h light:dark photoperiod.

Drosophila melanogaster transgenic strains used for imaging were obtained from the Bloomington Drosophila Stock Center (BDSC) (Bloomington, IN, US) and reared under the same conditions as described above.

\subsection{Drosophila suzukii ovipositor scanning electron microscopy}

Adult females of $D$. suzukii were anaesthetized by exposure to cold temperatures $\left(-18{ }^{\circ} \mathrm{C}\right)$ until death, then they were immediately soaked in $60 \%$ ethanol. The ovipositor of $D$. suzukii is positioned at the very tip of the abdomen, and, at rest, is held hidden within the last abdominal segments. With a gentle pressure on the abdomen it is possible to expose the ovipositor, which comprises two elongated sub-triangular plates ending in a tip. The ovipositor of each individual was dissected from the abdomen. Specimens were dehydrated in a series of graded ethanol, from $60 \%$ to $99 \%, 15 \mathrm{~min}$ for each step. After dehydration, $99 \%$ ethanol was substituted with pure HMDS (Hexamethyldisilazane, Sigma-Aldrich) and the specimens were allowed to dry under a hood at room temperature (RT); this step was repeated twice. Up to five samples were mounted on aluminium stubs, with different orientations, in order to obtain a clear view on the ventral and lateral sides of the ovipositor. Mounted specimens were gold-sputtered using a Balzers Union SCD 040 unit. The observations were carried out using a Philips XL 30 scanning electron microscope (SEM) operating at 7-10 KV, working distance 9-10 mm. 


\subsection{Drosophila suzukii ovipositor transmission electron microscopy}

Ten $D$. suzukii female individuals were anesthetized by exposure to cold temperatures $\left(-18{ }^{\circ} \mathrm{C}\right)$ for $60 \mathrm{~s}$, then immediately immersed in a solution of glutaraldehyde and paraformaldehyde (PFA) $2.5 \%$ in $0.1 \mathrm{M}$ cacodylate buffer ( $\mathrm{pH} 7.2-7.3$ ) plus $5 \%$ sucrose. The ovipositor was detached from the abdomen, reduced in size to help fixative penetration, and left at $4{ }^{\circ} \mathrm{C}$ for $24 \mathrm{~h}$. Then, the specimens were washed twice in cacodylate buffer for $10 \mathrm{~min}$, post-fixed in $1 \% \mathrm{OsO}_{4}$ for $1 \mathrm{~h}$ at $4{ }^{\circ} \mathrm{C}$, and rinsed in the cacodylate buffer. They were dehydrated in graded ethanol series from 60\% to $99 \%$ and embedded in Epon-Araldite with propylene oxide as bridging solvent. Thin sections were taken with a diamond knife on an LKB Bromma ultramicrotome and mounted on formvar-coated 50 mesh grids. Then, sections on grids were stained with uranyl acetate (20 min, RT) and with lead citrate (5 min, RT). Finally, the sections were imaged with a Philips EM 208 transmission electron microscopy (TEM). A digital camera MegaViewIII (SIS) provided high-resolution images.

\subsection{Drosophila suzukii ovipositor immunohistochemistry}

Drosophila suzukii adult females were anesthetized using $\mathrm{CO}_{2}$. Abdominal distal tips were cut with a razor blade and fixed in 4\% PFA in phosphate-buffered saline (PBS, pH 7.4) (Sigma-Aldrich) for $40 \mathrm{~min}$ on ice. Samples were then washed three times with PBS for $20 \mathrm{~min}$, incubated in 10\% sucrose (Sigma-Aldrich) solution, and kept rotating for $1 \mathrm{~h}$ at RT. Sucrose solution was increased to $25 \%$, and samples were kept rotating overnight at $4{ }^{\circ} \mathrm{C}$. Samples were then embedded in OCT (OCT mounting medium Q PATH, VWR), and mounted on a sample holder. Sections of $15 \mu \mathrm{m}$ thickness were cut with a CM 1510-3 cryostat (Leica) and collected on a SuperFrost glass slide (ThermoFisher Scientific). Slides were washed in PBS-T (PBS $+0.1 \%$ Triton-X-100, Sigma-Aldrich) for $5 \mathrm{~min}$, and then blocked with $5 \%$ normal goat serum (Sigma-Aldrich) in PBS-T for $30 \mathrm{~min}$. Anti-horseradish peroxidase (HRP) cyanine-conjugated antibodies (Cy3 AffiniPure Rabbit Anti-HRP, Jackson ImmunoResearch) diluted 1:300 were used to stain the neurons. Slides were kept in a moist chamber at $4{ }^{\circ} \mathrm{C}$ overnight in dark. The next day, antibodies were removed, and the slides were washed three times with PBS-T for 5 min and then mounted using Vectashield (Vector Laboratories).

\subsection{Examination of GAL4-driven GFP expression patterns in the D. melanogaster ovipositor}

The native GFP signal was observed at the level of the ovipositor of females expressing the super bright 6xGFP UAS-reporter (UAS-6xGFP; BDSC accession number 52262) under the pattern of the pan-neuronal nsyb-GAL4 driver (GMR57C10-GAL4; BDSC accession number 39171). Flies were anesthetized using $\mathrm{CO}_{2}$, abdominal distal tips were cut, embedded in $70 \%$ glycerol and immediately imaged.

\subsection{Confocal imaging}

Images were acquired using a Leica TCS SP8 confocal microscope, equipped with HC PL FLUOTAR 20x/0.55 DRY and HC PL APO CS2 $63 \mathrm{x} / 1.40$ OIL objectives. For D. suzukii, Cy3 excitation was performed using a 522-nm solid-state laser, and fluorescence was detected at 561-591 nm. Single images of $1024 \times 1024$ pixels (pixel size of $0.072 \times 0.072 \mu \mathrm{m}$ ) and stacks with z-steps of $2 \mu \mathrm{m}$ were acquired. For D. melanogaster, GFP excitation was performed using a $488-\mathrm{nm}$ solidstate laser, and fluorescence was detected at $499-524 \mathrm{~nm}$. Stacks of $1024 \times 1024$ pixels (pixel size of $0.06 \times 0.06 \mu \mathrm{m}$ ) optical section were generated with a z-interval of $2 \mu \mathrm{m}$. Images were analyzed using FijiImageJ software (Schindelin et al., 2012).

\subsection{RNA extraction and sequencing}

RNA was extracted from the terminalia of 3- to 10-day old mated females. Dissection was done with forceps and included both the genitalia and the analia (Supplementary Fig. S1). Dissected tissues were stored at $-80{ }^{\circ} \mathrm{C}$ in RNAlater (ThermoFisher Scientific) until extraction. Each species sample was composed of RNA extracted from around 60-80 individuals. Samples were homogenized using TissueLyser (Qiagen) and total RNA was extracted with TRIzol reagent (ThermoFisher Scientific), following the manufacturer's protocol. DNA contamination was removed with a DNase I (ThermoFisher Scientific) incubation step. A second RNA extraction with PureLink RNA Mini Kit (ThermoFisher Scientific) was performed to remove DNase and to concentrate samples. The total RNA $(\sim 1 \mu \mathrm{g} / \mathrm{sample})$ was sent to Beckman Coulter Genomics (Danvers, MA USA) for library preparation and Illumina sequencing. Library preparation was carried out through polyA + selection, and paired-end (PE) sequencing was run on an Illumina HiSeq 2500 System with V3 chemistry that generated $100 \mathrm{bp}$ reads. Raw reads are accessible from the Genbank SRA database (BioProject number PRJNA526247) (Supplementary Table S1).

\subsection{De novo transcriptome assembly, annotation, and gene ontology}

Raw reads were trimmed with Trimmomatic (Bolger et al., 2014). Both paired and unpaired reads were used for a de novo assembly of the transcriptome for each species with Trinity v2.0.6 (Grabherr et al., 2011), using the normalization step and flag -min_kmer_cov 2. The transcriptome quality was checked by mapping the paired reads against the assembled transcriptome with Bowtie2 with default parameters (Langmead and Salzberg, 2012). The four transcriptomes were annotated using Standalone Blast + . Blast searches were run with the command blastx using the predicted proteins from the $D$. melanogaster genome (version r6.25) as the database. The top hit for each sequence was retained when the E-value was less than $1 \times 10^{-10}$. PANTHER version 14.0 (Mi et al., 2017) was used to extract gene ontology (GO) terms (Panther GO-Slim) for each annotated transcriptome. Venn diagrams were created using Venny 2.1.0 (Oliveros, 2015).

Annotation of mechanosensory channels has been perfomed with iterative blastx searches against $D$. suzukii (Genbank accession number AWUT00000000) and D. biarmipes genomes (Genbank accession number AFFD00000000) using D. melanogaster sequences as query. The intron-exon structure was manually determined using BioEdit (Hall, 1999) and full-length coding sequences were extracted. Quantification of mechanosensory channels and chemoreceptor gene (which we previously annotated in D. suzukii and D. biarmipes, Crava et al., 2016; Ramasamy et al., 2016) expression was done with Bowtie2 with default parameters. For $D$. subpulchrella, whose genome is not available yet, we mapped the trimmed reads against the gene set from its sister-species $D$. suzukii. Transcript per million (TPM) were quantified by RSEM (Li and Dewey, 2011).

\subsection{Reverse transcription PCR of D. suzukii chemosensory-related genes}

Expression of chemosensory receptor genes in the D. suzukii terminalia identified by RNA-seq analysis was confirmed by reverse transcription PCR (RT-PCR). Orco and Gr64, which were not found to be expressed by RNA-seq, were used as negative control and genomic DNA as positive control. The used primers are listed in Supplementary Table S2. RNA was extracted with Trizol and treated with DNAse I as described before. $1 \mu \mathrm{g}$ RNA was then retrotranscribed to cDNA with SuperScript III Reverse Transcriptase (ThermoFisher Scientific) following the manufacturer's protocol. To control for genomic DNA contamination, RNA underwent a parallel mock reverse transcription step, in which the reverse transcriptase was omitted. Amplifications were carried out with GoTaq Green Master Mix (Promega) in a final volume of $25 \mu \mathrm{l}$ containing $1 \mu \mathrm{l}$ of cDNA diluted 1:10 and $0.4 \mu \mathrm{M}$ of each primer at 
the following conditions: $2 \mathrm{~min}$ at $95{ }^{\circ} \mathrm{C}$, then 25 cycles composed by a $30 \mathrm{~s}$ step at $95{ }^{\circ} \mathrm{C}, 30 \mathrm{~s}$ at $55{ }^{\circ} \mathrm{C}$, and $1 \mathrm{~min}$ at $72{ }^{\circ} \mathrm{C}$, followed by a final elongation step of $5 \mathrm{~min}$ at $72{ }^{\circ} \mathrm{C}$. PCR amplicons were run on $1 \%$ agarose gel stained with Midori Green Advance (Nippon Genetics).

\section{Results and discussions}

\subsection{Drosophila suzukii ovipositor carries four types of mechanosensilla-like structures}

The ovipositor of $D$. suzukii, as well as of its sister species $D$. subpulchrella, has been defined as "serrated" because of the presence of well-evident modified bristles, mostly arranged along its outer margin (i.e. the ventral side of the ovipositor, when considered in its resting position) (Supplementary Fig. 2) (Atallah et al., 2014). The length and the morphology of $D$. suzukii ovipositor were analysed in a comparative framework in previous studies (Atallah et al., 2014; Green et al., 2019). In particular, Atallah et al. (2014) described the external morphology and the number of bristles in D. suzukii and D. subpulchrella. Yet, ultrastructure observations or functional studies are lacking, and no information about the role these structures is known. Here we fill the gap analysing the modified bristles present in the distal tip of the ovipositor, which are thought to come into contact with the fruit flesh (Supplementary Fig. 2) (see Atallah et al., 2014 for bristle classification). Our observations reveal the presence of four types of cuticular elements: conical pegs type 1 (CP1, also defined modified lateral bristles by Atallah et al., 2014), conical pegs type 2 (CP2, also defined modified marginal bristles by Atallah et al., 2014), and two categories of previously undescribed apical sensilla: trichoid sensilla (TS), and chaetic sensilla (CS) (Fig. 2, Supplementary Fig. 2). The number, placement, and types of cuticular elements on drosophilids ovipositor valves vary greatly among species (Craddock et al., 2018) as well as the ovipositor size and shape (Atallah et al., 2014; Green et al., 2019). In general, Drosophila species seem to have a more or less regular row of sensilla or pegs distributed on the ventral side of each valve (also called thorn bristles in D. melanogaster, (Chen and Baker, 1997)) and a distinctive chaetic sensilla projecting from the ventral side of the ovipositor (Craddock et al., 2018). In D. melanogaster, this latter is referred to as the "long bristle" (Taylor, 1989a). Other features which are not displayed by all ovipositors are dorsal or lateral sensilla and microbristles or trichoidea sensilla into the ovipositor apex (Craddock et al., 2018; Taylor, 1989a).

Our observations estimate an average of $17 \mathrm{CP} 1 \mathrm{~s}(n=50$, $S D=0.99$ ) sitting on narrow sockets in the cuticle on the ventral margin of each ovipositor plate arranged in a single row. Their number is slightly higher than what is reported by Atallah et al (2014) in two different $D$. suzukii strains, suggesting that variation exists in number of these structures among individuals. Likewise, in D. melanogaster, the observed number of thorn bristles (the structures homologous to CP1s) varies from 11 to 17 (Chen and Baker, 1997; Taylor, 1989a). CP1 number did not differ significantly between $D$. suzukii and $D$. subpulchrella (Atallah et al., 2014). Our observations revealed that CP1s are about $15 \mu \mathrm{m}$ long with a base diameter of $10 \mu \mathrm{m}$ and are characterised by a cuticular shaft slightly bent towards the external side of the plate (Fig. 2A). The cuticle is grooved externally all along (Fig. 3A). Each structure ends in a sharp tip, although in some specimens the tip appears worn, having a blunt shape. The analysis of ultrathin sections shows that the internal structure is characterised by a solid, poreless

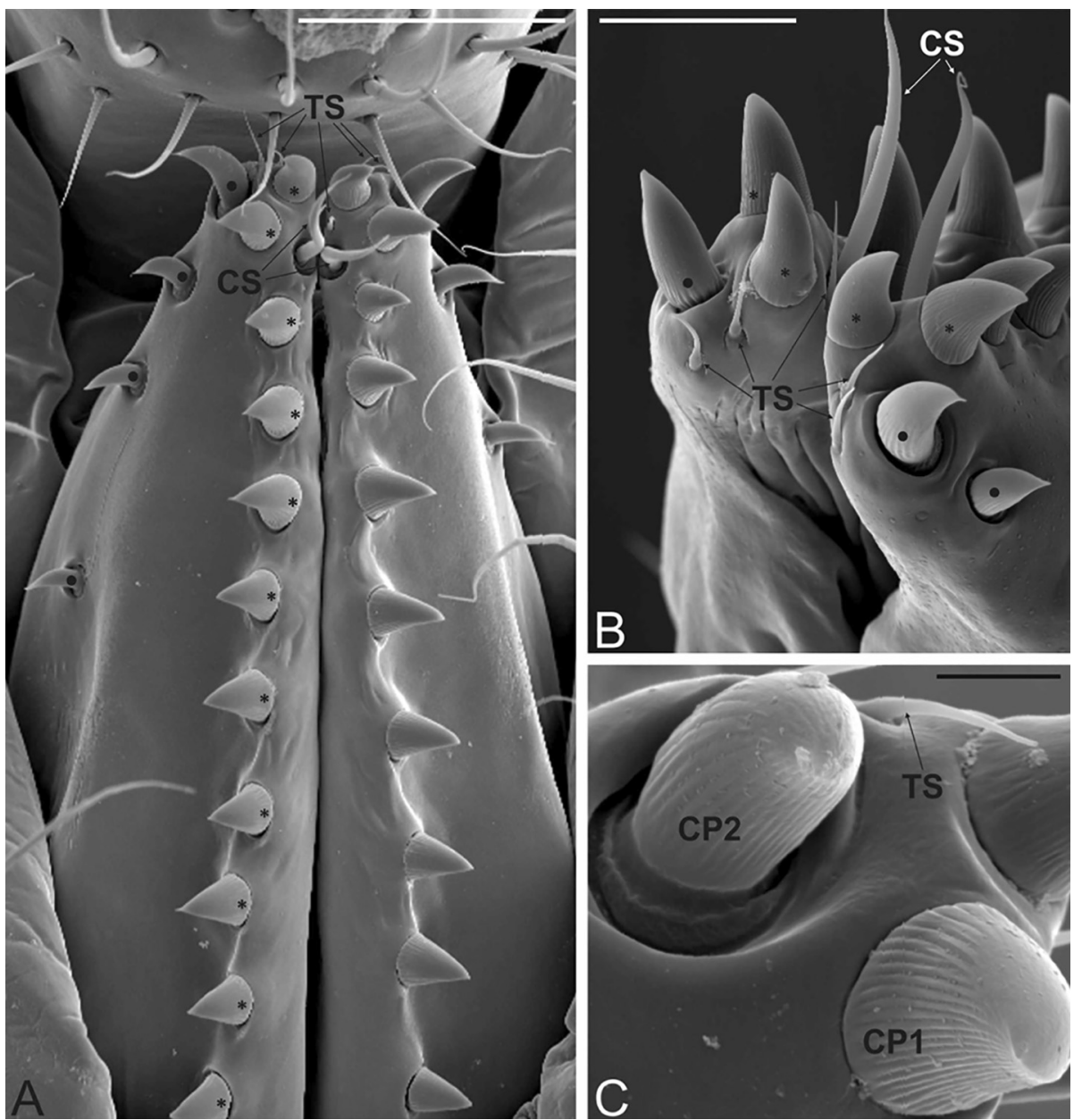

Fig. 2. Drosophila suzukii ovipositor pegs and sensilla (A) Ventral view of the ovipositor of $D$. suzukii showing the two ovipositor plates and the different structures that are present. The tip of each plate presents three trichoid sensilla (TS) and a chaetic sensillum (CS). A single row of conical pegs type 1 (*) is found, with the structures arranged along the ventral edge of each ovipositor plate. Four conical pegs type $2(\bullet)$ are present, with the first one sitting at the very tip of the ovipositor plate, while the others are positioned along a medial line of the ovipositor plate. (B) Detailed view of the tip of the ovipositor plates. The two apical TS are clearly visible, as well as the third, inserted just behind the most apical CP1. The CS are located very close to the TS. (C) Close-up view of the ovipositor plate tip. The $\mathrm{CP} 1$ is sitting on a narrow socket; it presents a grooved cuticle that smoothens at the tip. The CP2 is sitting on a large socket; it shows a grooved cuticle as well, but with less evident grooves and a pointed tip. Scale bars: A, $50 \mu \mathrm{m}$; B, $20 \mu \mathrm{m}$; C, $5 \mu \mathrm{m}$. 

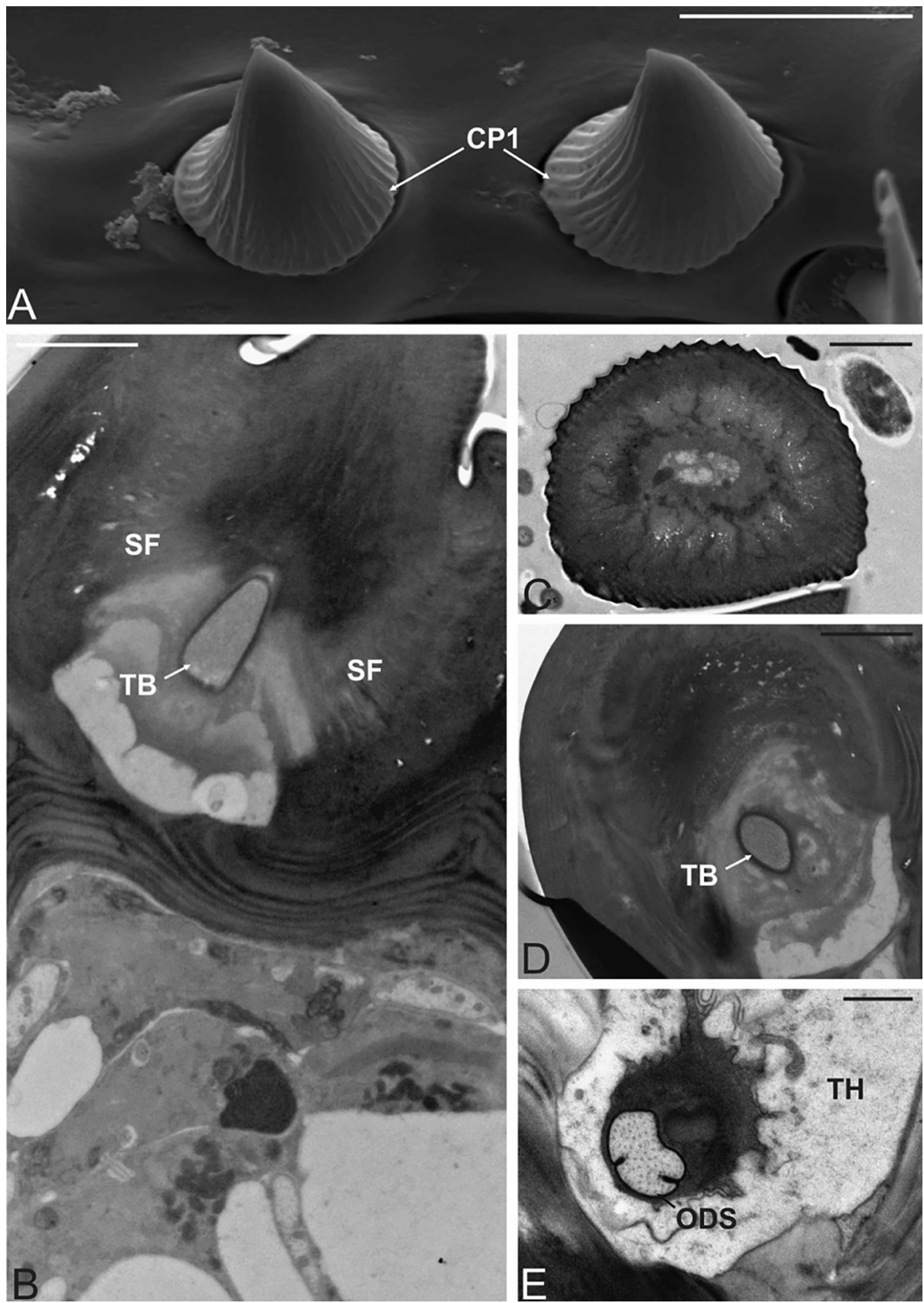

Fig. 3. Micrographs showing details of the conical pegs type 1 of the Drosophila suzukii ovipositor. (A) Scanning electron microscopy (SEM) ventral view of parts of the ovipositor plate ridge showing two conical pegs type 1 (CP1). (B) Transmission electron microscopy (TEM) longitudinal section at the socket level. The peg is sitting on a narrow socket made of thick cuticle. Suspension fibres (SF) are apparent, holding the peg and giving flexibility to the structure. The single sensory neuron associated with the CP1 terminates in a tubular body (TB) ending just at the base of the peg. (C-E) Serial TEM micrographs of a CP1 cross sections, taken at different levels, show the solid cuticular structure of the peg $(\mathrm{C})$, the presence of the tubular body (TB) at the socket level (D), and the outer dendritic segment (ODS) of the sensory neuron enclosed by the thecogen cell (TH) (E). Scale bars: A, $10 \mu \mathrm{m}$; B-D, $2 \mu \mathrm{m}$; E, $1 \mu \mathrm{m}$. cuticular shaft (Fig. 3B). Micrographs taken at the level of the medial peg show a thick and continuous cuticular wall with a small lumen without sensory neurons (Fig. 3C). Imaging at the socket level shows the presence of a single sensory neuron embedded in an electron-dense dendrite sheath, and ending in a tubular body (Fig. 3D and E). The tubular body is located at the base of the peg, where the socket with suspension fibres is evident (Fig. 3B). The tubular body is the site at the tip of the sensory dendrite where mechano-electrical transduction occurs (Erler, 1983; Keil and Steinbrecht, 1984; Marshall and Lumpkin, 2012). It is a feature typical of type I sensory neurons that construct cilia or flagella, which include bristle mechanoreceptors and propioreceptors such as chordotonal organs and campaniform sensilla (Gillespie and Walker, 2009; Kernan, 2007). All these evidences strongly suggest that $\mathrm{CP} 1 \mathrm{~s}$ are mechanosensilla.

Previous studies used the anti-HRP staining of the ventral thorn bristles of $D$. melanogaster (which correspond to D. suzukii CP1s) to provide evidences of their sensory nature (Taylor, 1989a,b). Labelling of D. suzukii ovipositor tip with anti-HRP is consistent with ultrastructure observations and shows the presence of a single sensory neuron that terminates at the base of each CP1 (Fig. 4). In D. melanogaster, we used the pan-neuronal marker $n$-syb to label thorn bristles and results show a single neuron ending at the base of each bristle, likewise to the anti-HRP staining in D. suzukii (Fig. 4). This anatomical similarity between $D$. suzukii and $D$. melanogaster suggests that also in the latter species, ventral ovipositor bristles could be mechanosensitive structures. Hence, CP1 function could be conserved in the ovipositor of Drosophila species independently of the ovipositor shape. This is consistent with results from Craddock et al. (2018) that did not identify any pore on the surface of ventral bristles in 41 Hawaiian drosophilids with dramatic differences in ovipositor morphology. However, further functional and structural studies are needed to rigorously verify this hypothesis in $D$. melanogaster and other Drosophila species.

Our observations reveal four to five CP2s arranged in line starting from the tip of each ovipositor plate (Fig. 2A). They are smaller than $\mathrm{CP} 1 \mathrm{~s}$ and show decreasing sizes from the most apical (14.5 $\mu \mathrm{m}$ long and $6 \mu \mathrm{m}$ of base diameter) to the proximal one (12.5 $\mu \mathrm{m}$ long and $3.5 \mu \mathrm{m}$ of 


\section{Drosophila suzukii}

\section{Bright Field}

A
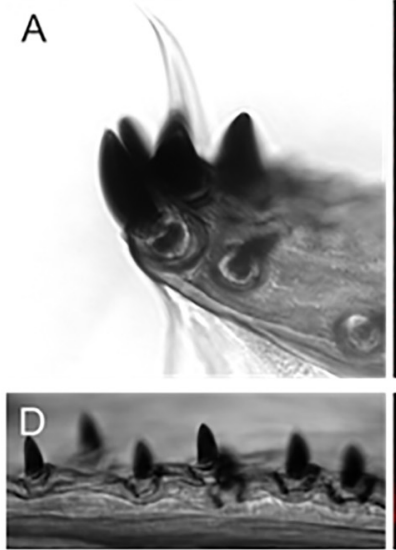
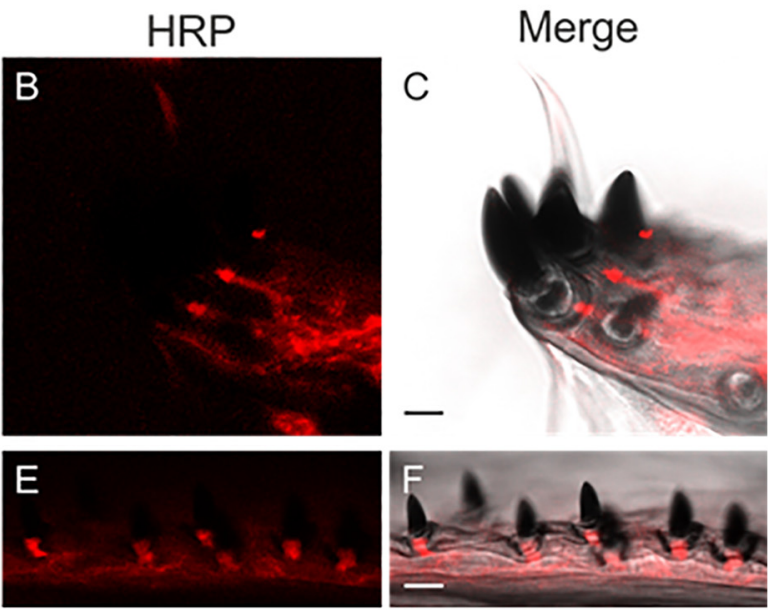

Merge

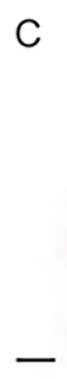

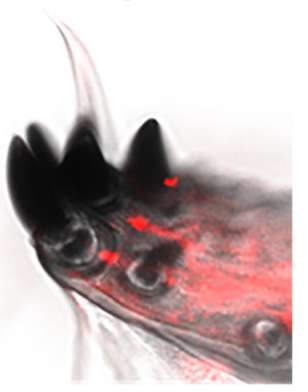

Fig. 4. Conical pegs are innervated by single neurons in both Drosophila suzukii and Drosophila melanogaster ovipositors Upper panel: Immunostaining of cryosection of the D. suzukii ovipositor plate: (A and D) bright-field, (B and E) counter staining with antihorseradish peroxidase (HRP) to visualize the neuron, ( $C$ and F) merged pictures. Scale bars: $10 \mu \mathrm{m}$. Lower panel: The pan-neuronal marker $n$-syb showed a single neuron innervating all ovipositor pegs in D. melanogaster. (A and D) bright-field, (B and $\mathrm{E}$ ) green fluorescent protein (GFP) visualization, (C and F) merged pictures. Scale bars: $20 \mu \mathrm{m}$. (For interpretation of the references to colour in this figure legend, the reader is referred to the web version of this article.)

\section{Drosophila melanogaster}
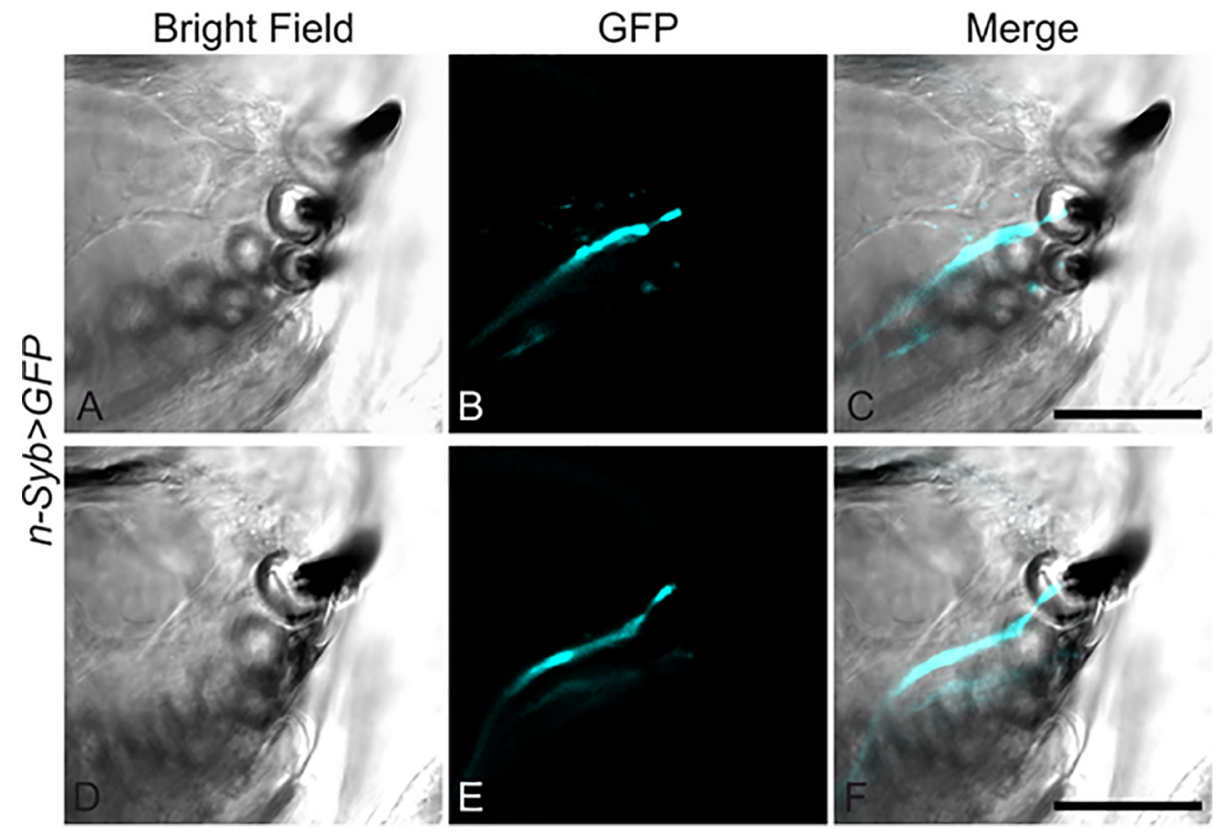

base diameter). Atallah et al. (2014) reported that size differences between CP1s and CP2s are less pronounced in D. subpulchrella than in $D$. suzukii, whereas in D. melanogaster no bristles equivalent to CP2s have been found (Taylor, 1989a). The cuticular shaft of CP2 is slightly grooved along the longitudinal axis for most of its length, although grooves are not as evident as in CP1 (Fig. 2C). Each CP2 ends in a fine tip that is absent in case of mechanical abrasion. The peg is sitting on an evident socket within the cuticular wall of the plate (Fig. 5A). TEM investigation revealed an internal structure similar to $\mathrm{CP} 1$, i.e. the presence of a solid cuticular shaft, devoid of pores (see inset in Fig. 5A), a small internal lumen without sensory neurons, and a single sensory neuron with a distal tubular body attached at the base of the peg (Fig. 5B-D). The peg itself is attached flexibly to the cuticle through a large socket with an abundance of suspension fibres (Fig. 5B). Anti-HRP staining highlights the presence of a single sensory neuron that stops at the base of each CP2 (Fig. 4). All these evidences suggest that CP2s are mechanosensitive structures likewise CP1s.

At the apex of each $D$. suzukii ovipositor plate, there are three small TS (Fig. 2B). Two are located on the dorsal side of the plate, whereas the third one is located apically (Fig. 6A). In D. melanogaster three trichoid ovisensilla have also been reported (Taylor, 1989a). In D. suzukii, they are slender and finely tipped sensilla with a smooth cuticular shaft devoid of cuticular pores (12.5 $\mu \mathrm{m}$ long and $1.5 \mu \mathrm{m}$ of base diameter). These sensilla are sitting in the cuticular wall on distinct sockets, running almost parallel to the plate cuticular wall itself. TEM images reveal that TS are made of solid cuticle, there are no pores on the cuticle and no sensory neurons entering the peg lumen (Fig. 6B). A single sensory neuron is associated with each TS, reaching the sensillum base through a distal tubular body (Fig. 6C and Supplementary Fig. S3). In D. melanogaster, these three trichoidea sensilla appeared innervated by multiple neurons and have been hypothesized to be endowed with chemosensory functions (Taylor, 1989b). However, our observations in $D$. suzukii refute a role in chemosensation.

Each ovipositor plate shows the presence of a single CS inserted into the inner face of each valve tip. It is long $(38 \mu \mathrm{m})$ and slender $(2.5 \mu \mathrm{m}$ of base diameter), with a typical curved shape and a very fine tip (Fig. 6A). It is sitting on a large socket in the plate wall. Externally, the CS wall is smooth. Serial ultrathin sections revealed that the CS 


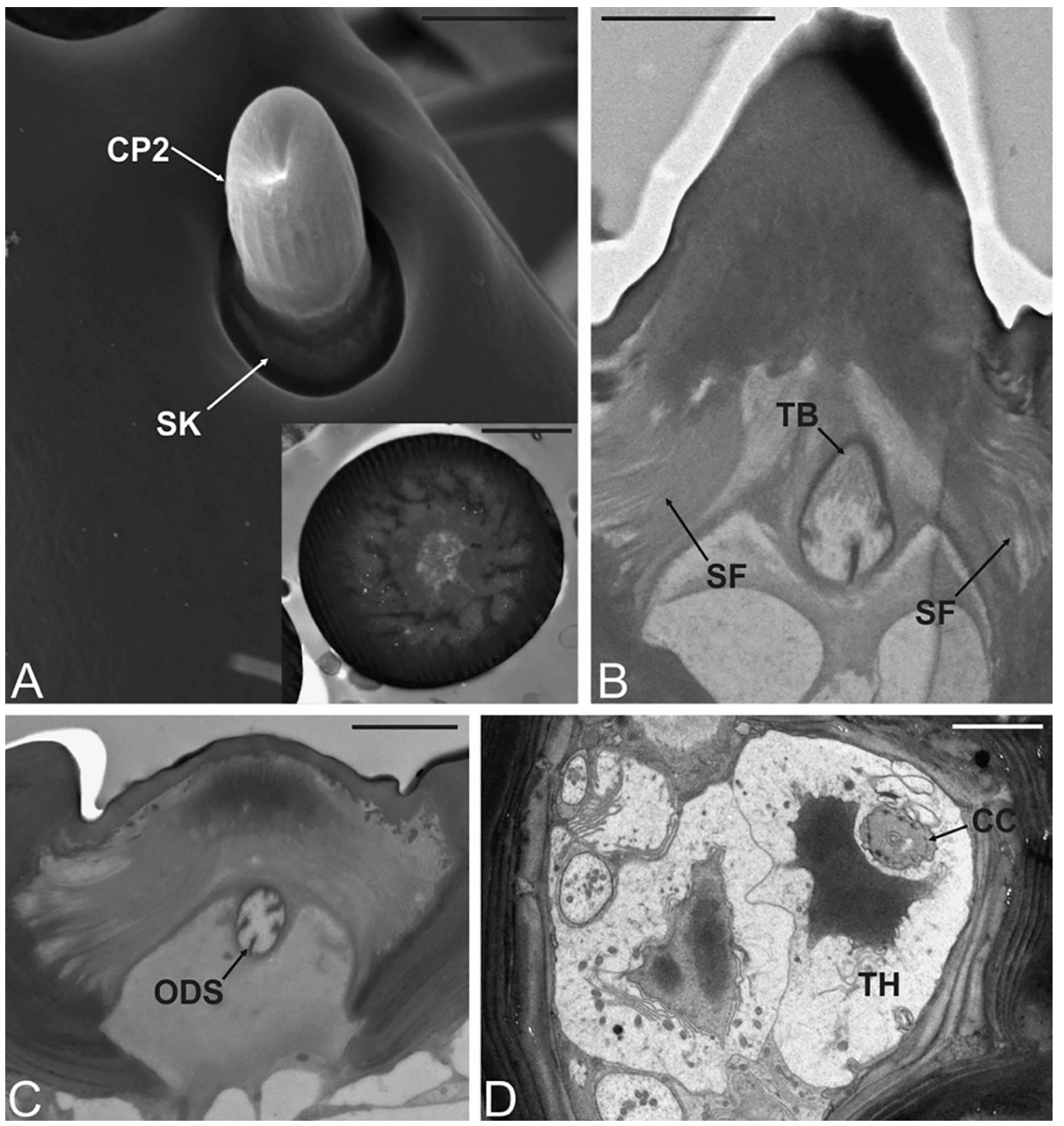

Fig. 5. Micrographs showing details of the conical pegs type 1 of the Drosophila suzukii ovipositor (A) SEM ventral view of the ovipositor plate, showing one of the conical pegs type 2 (CP2), with a slightly grooved cuticle and a sharp tip. Noticeable is also the large socket (SK) on which the CP2 is sitting in the cuticular wall of the ovipositor plate. The inset in (A) shows the TEM micrograph of a CP2 cross section, taken at half of the length of the peg: the peg is made of solid, thick cuticle and presents a reduced lumen, devoid of sensory neurons. (B-D) Serial TEM micrographs of a CP2, longitudinally and cross sections taken at different levels. They show in (B) the base of a CP2 with a large flexible socket and several suspension fibres (SF). The single sensory neuron ends in a tubular body (TB) at the base of the peg. In (C) the large socket is visible, as well as the outer dendritic segment (ODS) of the sensory neuron. In (D) a cross section is imaged at a lower level respect to the previous: the sensory neuron appears at the ciliary constriction level (CC), and it is enclosed by the thecogen cell (TH). Scale bars: A, $5 \mu \mathrm{m}$; inset in A, $2 \mu \mathrm{m}$; B-D, $2 \mu \mathrm{m}$.

cuticular shaft is made of solid cuticle and shows a central lumen without neurons (Fig. 6D). At the base, the shaft is attached to the cuticle and suspended through an elaborated socket with numerous suspension fibres (Fig. 6E). A single sensory neuron ends in a tubular body that attaches at the sensillum base (Fig. 6F). This is a typical feature of mechanosensitive-like organs (Erler, 1983; Keil and Steinbrecht, 1984; Tuthill and Wilson, 2016), and together with the absence of pores on the surface of the $D$. suzukii CS clearly points out a likely function in mechanotransduction. However, in two groups of Hawaiian Drosophila species, the apical CS (referred to as "long subapical sensillum") is uniporous, hence suggesting that in some species it may have taste function that helps to exploit specific ecological microniches (Craddock et al., 2018).

\subsection{RNA-seq characterization of the terminalia of four Drosophila species}

Since variation in egg-laying sites and ovipositor morphology among Drosophila species exists, we were interested in determing whether there is a corresponding level of variation in gene-expression. To this purpose, we compared de novo assembled transcriptomes from the terminalia of four Drosophila species with different ovipositor shapes and egg-laying behaviors (Fig. 1). Female terminalia can be divided in two parts, genitalia and analia. The genitalia comprise the external ovipositor plates and the internal ducts of the reproductive system, whereas the analia are made by the external anal plates and the posterior hindgut (Taylor, 1989a). Thus, our RNA-seq analysis reflects transcripts expressed in all of these tissues.

Illumina RNA-seq libraries from the terminalia of four Drosophila species generated an average of $60 \mathrm{M}$ ( $\pm 2.4 \mathrm{M} \mathrm{SD}) 100 \mathrm{bp}$ paired-end reads that led to four de novo assembled transcriptomes with contig counts ranging from 31,315 (D. subpulchrella) to 40,162 (D. suzukii) (Supplementary Table S1). Although reference genomes for three out of four Drosophila species studied were available, in the present study, transcripts were de novo assembled instead of mapping to genomic sequences because of the dramatic differences in genome completeness and resolution that would affect the detection of several transcripts. Our strategy has yelded four assemblies of comparable number of contigs $(34,884 \pm 4,017)$, N50 $(2,728 \pm 4,588)$ and percentage of reads successfully aligned to the assemblies $(80 \pm 6)$ (Supplementary Table S1). Thus, they are appropriate for a comparative analysis aimed to study transcript composition among assemblies and presence or absence of specific transcripts. We used blastx to identify homologous genes in D. melanogaster and assign gene ontology (GO) terms to our contigs. On average, $70 \%$ of contigs from each of the four transcriptomes have a blastx hit against the $D$. melanogaster predicted proteome (Supplementary Datase S1). Unique hits retrieved for each species vary from 8537 (D. subpulchrella) to 9634 (D. melanogaster). Of these, 7294 hits are common among the four Drosophila species, and represent the conserved transcriptional core for the Drosophila terminalia (Fig. 7A). GO analysis shows that the common terminalia genes are mostly involved in cellular processes (32\%), metabolic processes (29\%), and biological regulation (15\%) (Fig. 7B). Species-specific hits are few, namely 163 for $D$. suzukii, 131 for $D$. subpulchrella, 187 for $D$. biarmipes, and 682 for $D$. melanogaster, respectively. Unique D. suzukii hits have 811 unique GO terms (Fig. 7C, and Supplementary Datase S2). Among them there are contigs homologous to genes involved in pigmentation, cellular component organization, and response to stimulus compared to the other species (Fig. 7D). Contigs annotated with 

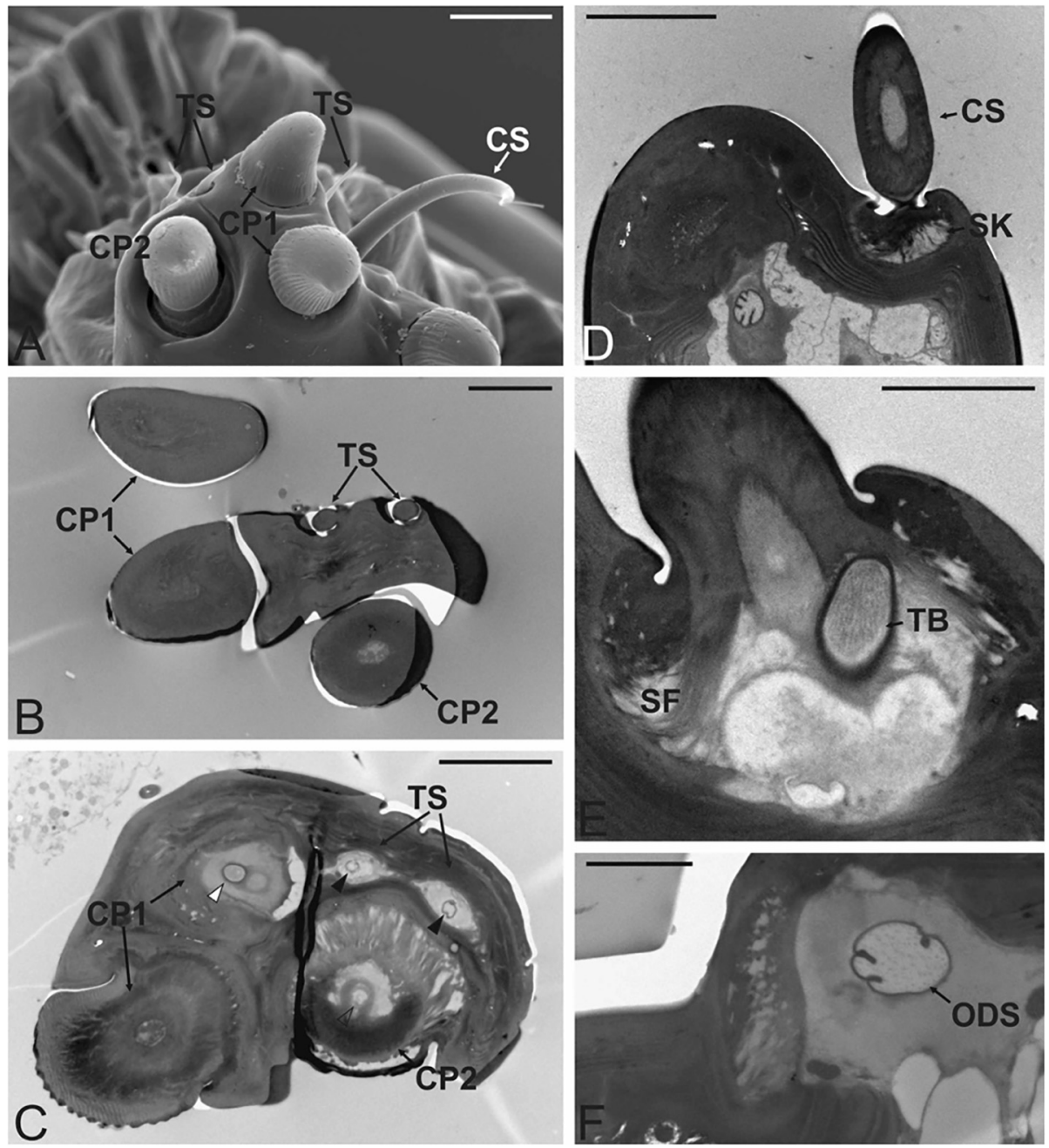

Fig. 6. Micrographs showing details of the trichoid and chaetic sensilla. (A) Scanning electron microscopy (SEM) picture of the ovipositor plate tip showing the three trichoid sensilla (TS) and the single chaotic sensillum (CS). (B, C) Serial transmission electron microscopy (TEM) cross sections of the ovipositor plate. In (B) the section is taken most apically and shows the two TS made of solid cuticle entering the cuticular wall, no sensory neurons were detected at this level. In (C) the two TS are pictured more proximally, the peg is no longer visible but two sensory neurons (one per each TS) are visible (black arrowheads). White and blank arrowheads show the sensory neurons associated with conical pegs type 1 (CP1) and type 2 (CP2), respectively. (D-F) Serial TEM longitudinal sections showing the main ultrastructural features of a CS: in (D) the CS is taken at the socket level (SK) and shows a thick cuticle with a central lumen without sensory neurons; in (E) a single sensory neuron ending in a tubular body (TB) inserted at the CS base is visible. The socket presents numerous suspension fibres (SF). In (F) the outer dendritic segment (ODS) of the sensory neuron is visible. Scale bars: A, $10 \mu \mathrm{m}$; B-C-D, $5 \mu \mathrm{m}$; E-F, $2 \mu \mathrm{m}$. pigmentation are homologous to yellow-g2. Proteins belonging to yellow gene family are involved in the synthesis of melanic pigment (Ferguson et al., 2011; Gompel et al., 2005), which may be related to the phenotypic plasticity of $D$. suzukii, whose winter morph phenotype is characterized by darker pigmentation (Shearer et al., 2016). Contigs annotated to cellular component organization are homologous to genes related to ribosome biogenesis. The ovipositor of $D$. suzukii has bigger ovipositor cells than D. melanogaster (Green et al., 2019), and this expression of contigs related to ribosome biogenesis may reflect the cost of cell maintenance.

\subsection{Conserved set of transcripts associated with mechanotransduction in Drosophila terminalia}

Given the ultrastructure analysis revealed that $D$. suzukii ovipositor pegs and sensilla are mechanosensitive-like structures, we were interested in determining which mechanosensitive channels and other sensory-related transcripts are expressed in terminalia. This may give an hint of the genes expressed in the mechanosensory-like structures highlighted in ultrastructure analysis; however it is worth to note that terminalia comprises both genitalia and analia, and also this last structure might carry mechanosensory-like structures. Detailed ultrastructure studies of analia has not been addressed in any Drosophila species used for RNA-seq, however in D. melanogaster some sensory structures whose likely function remains unknown have been observed in analia and in the eight tergite, which wraps the anal plate (Taylor, 1989a). We specifically looked for contigs orthologous to mechanosensitive genes described so far in D. melanogaster (Karkali and Martin-
Blanco, 2017): the putative metazoan mechanotransduction channels, i.e. degenerin/epithelial $\mathrm{Na}^{+}$channel C (DeG/eNaC) (Adams et al., 1998; Gorczyca et al., 2014; Guo et al., 2014; Jang et al., 2019; Tsubouchi et al., 2012; Zhong et al., 2010), the transient receptor potential (TRP) channels (Cheng et al., 2010; Gong et al., 2004; Göpfert et al., 2006; Tracey et al., 2003; Tsubouchi et al., 2012; Walker et al., 2000), the two-pore domain $\mathrm{K}^{+}$channel proteins (K2P) (Tabarean and Morris, 2002), as well as piezo (Kim et al., 2012), piezo-like (pzl) (Hu et al., 2019) and transmembrane channel-like $(t m c)$ genes (Guo et al., 2016).

Consistent with the results of the ultrastructural analysis, a conspicuous number of mechanosensitive-related transcripts are present in the $D$. suzukii assembly (Fig. 8). All the candidate mechanosensitive genes present in $D$. suzukii terminalia transcriptome are commonly present in more than one of the other Drosophila assemblies (Fig. 8A), pointing out that mechanosensation is a feature equally present in the terminalia of all species. Among genes whose expression is completely shared among the four species there are three DeG/eNaC proteins (which in Drosophila are commonly referred to as pickpocket proteins): $p p k, r p k$, and ppk26 (Adams et al., 1998; Gorczyca et al., 2014; Tsubouchi et al., 2012; Zhong et al., 2010). Only transcripts encoding a fourth pickpocket protein (ppk30), which has been recently related to mechanotransduction in D. melanogaster (Jang et al., 2019), are absent in all samples. Also piezo, its paralog pzl, as well as shaker and painless (pain) have a presence-absence pattern conserved across species. The four assemblies contain contigs homologous to piezo, that is a transmembrane protein involved in mechanosensitive nociception in $D$. melanogaster (Kim et al., 2012), but not to pzl. Contigs homologous to 
A

D. biarmipes

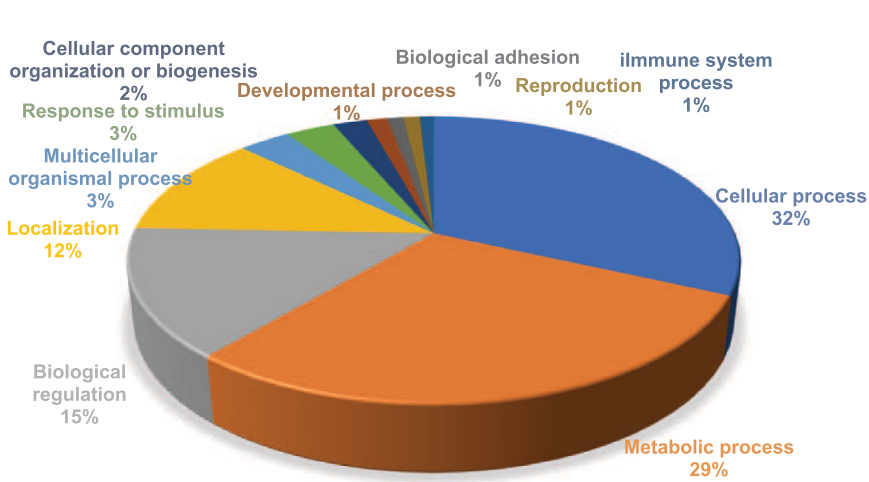

C

\begin{tabular}{lcc} 
Species & \#unique hits & \# GO terms \\
\hline D. suzukii & 163 & 811 \\
D. subpulchrella & 131 & 683 \\
D. biarmipes & 187 & 958 \\
D. melanogaster & 682 & 3481
\end{tabular}

D

\section{Cellular process}

Metabolic process

Biological regulation

Localization

Multicellular organismal process

Response to stimulus

Cellular component organization

Biological adhesion

Developmental process

Reproduction

Immune system process

Biological phase

Pigmentation

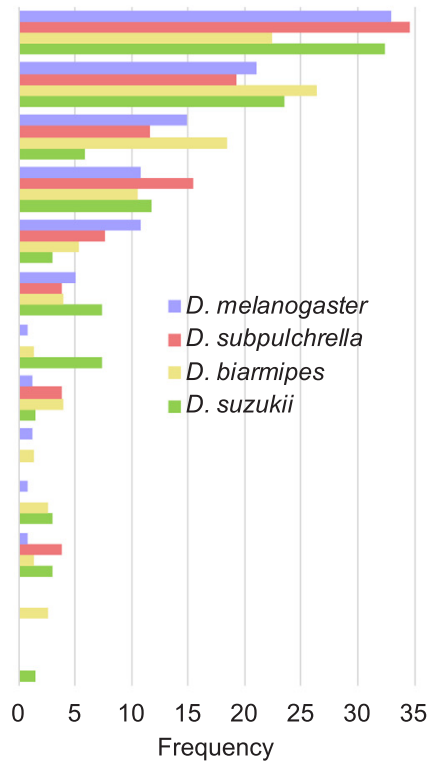

Fig. 7. Annotation of transcriptomes from the terminalia of four Drosophila species. (A) Venn diagram representing the unique D. melanogaster gene hits retrieved by blastx searches using contigs from each assembly as query. (B) Gene Ontology (GO) classification for the 7294 gene hits common to the four assemblies referred to Biological Process (Panther GO-Slim terms). (C) Number of species-specific hits and associated GO terms (D) GO classification for species-specific hits referred to Biological Process (Panther GO-Slim terms). Abbreviations: Dmel, D. melanogaster; Dbia, D. biarmipes; Dsuz, D. suzukii; Dsubp, D. subpulchrella.

A
\begin{tabular}{|l|l|l|}
\hline Gene symbol & D. suzukii assembly & Other Drosophila assemblies \\
\hline Degenerin/epithelial Na+ channel C (DeG/eNaC) \\
\hline$p p k$ & Present & Present \\
$r p k$ & Present & Present \\
ppk26 & Present & Present \\
ppk30 & Absent & Absent \\
\hline Transient receptor potential (TRP) channels \\
\hline pain & Present & Present \\
nompC & Present & D. melanogaster \\
iav & Present & D. biarmipes, D. melanogaster \\
nan & Absent & D. biarmipes \\
\hline Two-pore domain K+channel proteins (K2P) \\
\hline Sh & Present & Present \\
\hline Piezo proteins & \multicolumn{3}{|l|}{} \\
\hline Piezo & Present & Present \\
Pzl & Absent & Absent \\
\hline Transmembrane channel-like (TMC) proteins \\
\hline Tmc & Absent & D. biarmipes, D. melanogaster \\
\hline
\end{tabular}
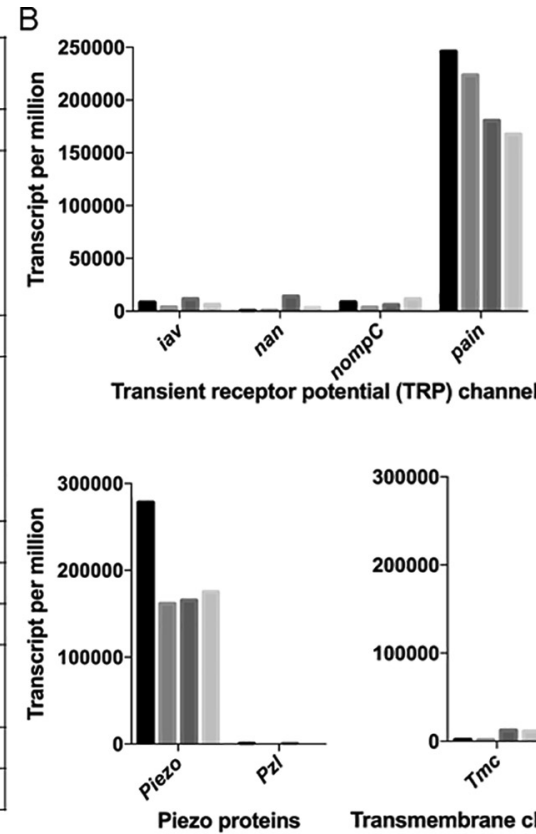

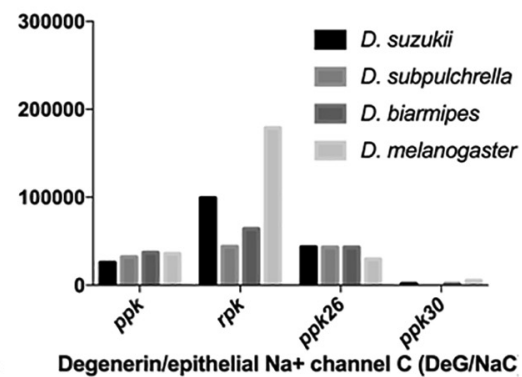

Degenerin/epithelial $\mathrm{Na}+$ channel C (DeG/NaC

Fig. 8. Annotation of transcriptomes from the terminalia of four Drosophila species. (Left panel) Table highlighting the presence or absence of contigs orthologous to Drosophila melanogaster mechanosensitive genes in D. suzukii de novo transcriptome assembly and in the other three assemblies (Right panel) Quantification of mRNA levels of mechanosensitive genes in $D$. melanogaster, $D$. suzukii, $D$. biarmipes and $D$. subpulchrella terminalia. Trimmed reads were mapped against manually annotated mechanosensitive genes except for $D$. subpulchrella, whose reads were mapped against the sister-species $D$. suzukii annotation set. 
shaker, which is a K2P stretch-sensitive ion channel (Tabarean and Morris, 2002), are present in the four assemblies as well as contigs homologous to pain, which codes for a TRP channel required for both thermal and mechanical nociception (Tracey et al., 2003).

In a subset of assemblies there are transcripts encoding tmc and TRP channels other than pain involved in mechanotransduction. tmc is expressed in $D$. melanogaster larval peripheral sensory neurons and it is involved in proprioception and sensory control of larval locomotion (Guo et al., 2016). Contigs homologs to this gene are present only in $D$. melanogaster and D. biarmipes assemblies, while they are absent in transcriptomes from species with serrated ovipositors. TRP proteins are membrane proteins that mediate many forms of sensory perception, including mechanosensation. Among TRPs involved in mechanotransduction, no mechanoreceptor potential $C$ (nompC), which is the bona fide mechanotransduction channel in Drosophila (Walker et al., 2000 ) is present only in D. suzukii and D. melanogaster assemblies. Contigs homologous to inactive (iav) are absent in D. subpulchrella assembly, and contigs homologous to nanchung (nan) are present only in the $D$. biarmipes assembly. Since iav and nan encode interdependent channel subunits and require each other for expression in D. melanogaster (Gong et al., 2004), we sought to understand why we found transcripts corresponding to only one of the two transcripts in the assemblies of $D$. suzukii and D. melanogaster. One possibility is that their expression is localized in a small number of cells and that their presence has been diluted by analysing a composite heterogeneous tissue such as the terminalia (Johnson et al., 2013). To explore if the absence of some transcripts is due to artifacts, we quantified expression of manually annotated mechanosensitive-related genes in each species (Fig. 8B, Supplementary Datase S3). Although the absence of replicates does not allow us to study in depth species-specific expression patterns, expression levels may indicate if a specific gene has similar or dramatically different mRNA levels among the four species. Our data reveal that all genes showing an inconsistent presence-absence pattern across species (iav, nan, nompC and $t m c$ ) have a low expression signal in all Drosophila; thus their absence from some de novo assembled transcriptome may be due to limiting number of reads available for assembling. Interestingly, likewise genes with an inconsistent presence-absence pattern, also genes present in the four assemblies have similar mRNA levels shared across species.

\subsection{Expression of chemosensory-related genes in Drosophila terminalia}

The four assemblies contain contigs homologous to genes involved in chemosensory perception, i.e. several gustatory receptors (GRs), ionotropic receptors (IRs), and odorant receptors (ORs) (Supplementary Table S3). In particular, contigs homologous to Ir47a are present in all four assemblies, and contigs homologous to Ir62a and Gr66a are present in three out of four assemblies. Interestingly, these three genes are among the most expressed ones in all species (Supplementary Fig. S4). Among the other highest expressed chemoreceptor genes there is Or43b. Surprisingly, we did not find any trace of the olfactory receptor co-receptor (Orco) expression, which is required to have functional odorant receptors (Joseph and Carlson, 2015). In D. suzukii, Orco absence has been confirmed by RT-PCR (Supplementary Fig. S5).

The genomes of $D$. suzukii or $D$. biarmipes contain several paralogous genes that encode chemoreceptors and are not shared with $D$. melanogaster (Crava et al., 2016; Ramasamy et al., 2016). Gene duplication may be source of novel properties, e.g. paralogs may be expressed in body parts other than the ancestral expression site where they take care of new functions (Kaessmann, 2010). No paralogous genes are present in $D$. suzukii and $D$. biarmipes terminalia transcriptomes, with the exception of $D$. suzukii Ir52f2, but its expression could not be validadet by RT-PCR (Supplementary Figure S5). These results point out that duplicated chemoreceptor genes identified in these two species do not carry out any new function in terminalia.

Expression of chemoreceptor genes in D. suzukii terminalia should not be related to the presence of sensilla and pegs on the tip of the ovipositor valves, since ultrastructure analysis detailed features compatible only with mechanosensitive-like organs. However, chemosensory structures may be present in other tissues in the terminalia, such as the analia (Supplementary Fig. S1). In D. melanogaster, analia carry several small and long bristles. In addition, the eight tergite, which wraps the anal plate, is scattered with thin bristles (Taylor, 1989a). Moreover, it has been shown that, besides the main function in taste, some GRs (and likely some IRs) may have other non-gustatory functions, such as the detection of internal ligands. For example, internal sensory neurons are present in the reproductive tract of $D$. melanogaster to sense sex peptide (Lee et al., 2016; Naccarati et al., 2012), and this may be a location where chemoreceptor transcripts are expressed. Gr43a, which is involved in fructose detection in the D. melanogaster brain, is also expressed in the female uterus, possibly for the detection of fructose in the seminal fluid (Sato et al., 2011). Gr43a was found only in D. biarmipes assembly, and its expression levels in the four species are quite low, possibly due to an expression pattern limited to few cells.

\section{Conclusions}

Our results represent the first step towards a full molecular, anatomical, and physiological characterization of sensory perception in the ovipositor of $D$. suzukii. In particular, these findings show that (i) pegs and sensilla housed in the tip of the $D$. suzukii ovipositor tip have a mechanosensilla-like structure, (ii) indicate that such feature may be shared with $D$. melanogaster, a species with blunt-end ovipositor, (iii) provide a qualitative overview of genes expressed in the terminalia of four Drosophila species with different ovipositor shapes. We propose that the sensilla and pegs present on the ovipositor tip of $D$. suzukii are the sensory structures responsible to probe substrate stiffness for the egg-laying site selection. It is also possible that these sensory structures work together with other sensory organs in assessing when the ovipositor has penetrated the substrate and peristaltic waves can start pushing down the egg. We also suggest that ovipositor mechanosensitive-like organs are likely widespread across Drosophila species independently of the ovipositor shape. Future functional studies will clarify the relationship between the structure and the function of the ovipositor sensilla.

Previous research has demonstrated that fruit stiffness is the crucial component in host selection and is negatively related to oviposition and, as a consequence, fruit susceptibility to D. suzukii (Baser et al., 2018; Burrack et al., 2013; Ioriatti et al., 2015; Kinjo et al., 2013; Lee et al., 2011). In this wider context, our work provides a necessary starting point to elucidate the molecular and physiological basis of the mechanotransduction system in the ovipositor of $D$. suzukii, which might allow for the development of mechanotransduction-based control strategies.

\section{Acknowledgements}

Cristina M. Crava has been a recipient of a FP7-PEOPLE- 2013-IEF grant from the European Union (grant ID 627755). This research was funded by the Italian Ministery of Education, University and Research (program MIUR-FFABR 2017) to Albrecht Haase. We would like to thank Elisabetta Leonardelli for Drosophila rearing and laboratory assistance.

\section{Appendix A. Supplementary data}

Supplementary data to this article can be found online at https:// doi.org/10.1016/j.jinsphys.2020.104088.

\section{References}

Adams, C., Anderson, M.G., Motto, D., Price, M., Johnson, W.A., Welsh, M.J., 1998. 
Ripped pocket and pickpocket, novel Drosophila DEG/ENaC subunits expressed in early development and in mechanosensory neurons. J. Cell Biol. 140, 143-152.

Asplen, M.K., Anfora, G., Biondi, A., Choi, D.-S., Chu, D., Daane, K.M., Gibert, P., Gutierrez, A.P., Hoelmer, K.A., Hutchison, W.D., Isaacs, R., Jiang, Z.-L., Kárpáti, Z., Kimura, M.T., Pascual, M., Philips, C.R., Plantamp, C., Ponti, L., Vétek, G., Vogt, H., Walton, V.M., Yu, Y.i., Zappalà, L., Desneux, N., 2015. Invasion biology of spotted wing Drosophila (Drosophila suzukii): a global perspective and future priorities. J. Pest. Sci. 88 (3), 469-494. https://doi.org/10.1007/s10340-015-0681-z.

Atallah, J., Teixeira, L., Salazar, R., Zaragoza, G., Kopp, A., 2014. The making of a pest: the evolution of a fruit-penetrating ovipositor in Drosophila suzukii and related species. Proc. R. Soc. B Biol. Sci. 281, 20132840. https://doi.org/10.1098/rspb.2013. 2840.

Baser, N., Broutou, O., Verrastro, V., Porcelli, F., Ioriatti, C., Anfora, G., Mazzoni, V., Rossi Stacconi, M.V., 2018. Susceptibility of table grape varieties grown in south-eastern Italy to Drosophila suzukii. J. Appl. Entomol. 142, 465-472. https://doi.org/10. 1111/jen.12490.

Bolger, A.M., Lohse, M., Usadel, B., 2014. Trimmomatic: A flexible trimmer for Illumina sequence data. Bioinformatics 30, 2114-2120. https://doi.org/10.1093/ bioinformatics/btu170.

Burrack, H.J., Fernandez, G.E., Spivey, T., Kraus, D.A., 2013. Variation in selection and utilization of host crops in the field and laboratory by Drosophila suzukii Matsumara (Diptera: Drosophilidae), an invasive frugivore. Pest Manag. Sci. 69, 1173-1180 https://doi.org/doi:10.1002/ps.3489.

Chen, E.H., Baker, B.S., 1997. Compartmental organization of the Drosophila genital imaginal discs. Development 124, 205-218.

Cheng, L.E., Song, W., Looger, L.L., Jan, L.Y., Jan, Y.N., 2010. The role of the TRP channel NompC in drosophila larval and adult locomotion. Neuron 67, 373-380. https://doi. org/10.1016/j.neuron.2010.07.004.

Cini, A., Ioriatti, C., Anfora, G., 2012. A review of the invasion of Drosophila suzukii in Europe and a draft research agenda for integrated pest management. Bull. Insectol. 65, 149-160.

Cloonan, K.R., Abraham, J., Angeli, S., Syed, Z., Rodriguez-Saona, C., 2018. Advances in the chemical ecology of the spotted wing Drosophila (Drosophila suzukii) and its applications. J. Chem. Ecol. 44, 922-939. https://doi.org/10.1007/s10886-0181000-y.

Craddock, E.M., Kambysellis, M.P., Franchi, L., Francisco, P., Grey, M., Hutchinson, A., Nanhoo, S., Antar, S., 2018. Ultrastructural variation and adaptive evolution of the ovipositor in the endemic Hawaiian Drosophilidae. J. Morphol. 279, 1725-1752. https://doi.org/10.1002/jmor.20884.

Crava, C.M., Ramasamy, S., Ometto, L., Anfora, G., Rota-Stabelli, O., 2016. Evolutionary insights into taste perception of the invasive pest Drosophila suzukii. G3 Genes Genomes Genet. 6, 4185-4196. https://doi.org/10.1534/g3.116.036467.

Erler, G., 1983. Reduction of mechanical sensitivity in an insect mechanoreceptor correlated with destruction of its tubular body. Cell Tissue Res. 234, 451-461.

Falk, R., Atidia, J., 1975. Mutation affecting taste perception in Drosophila melanogaster. Nature 254, 325-326.

Falk, R., Bleiser-Avivi, N., Atidia, J., 1976. Labella taste organs of Drosophila melamogaster. J. Morphol. 150, 327-341.

Ferguson, L.C., Green, J., Surridge, A., Jiggins, C.D., 2011. Evolution of the insect yellow gene family. Mol. Biol. Evol. 28, 257-272. https://doi.org/10.1093/molbev/msq192.

Gillespie, P.G., Walker, R.G., 2009. Mechanotransduction by hair cells: models, molecules, and mechanisms. Nature 139, 33-44.

Gompel, N., Prud'homme, B., Wittkopp, P.J., Kassner, V.A., Carroll, S.B., 2005. Chance caught on the wing: cis-regulatory evolution and the origin of pigment patterns in Drosophila. Nature 433, 481-487.

Gong, Z., Son, W., Chung, Y.D., Kim, J., Shin, D.W., McClung, C.A., Lee, Y., Lee, H.W., Chang, D.J., Kaang, B.K., Cho, H., Oh, U., Hirsh, J., Kernan, M.J., Kim, C., 2004. Two interdependent TRPV channel subunits, inactive and nanchung, mediate hearing in Drosophila. J. Neurosci. 24, 9059-9066. https://doi.org/10.1523/JNEUROSCI.1645 04.2004.

Göpfert, M.C., Albert, J.T., Nadrowski, B., Kamikouchi, A., 2006. Specification of auditory sensitivity by Drosophila TRP channels. Nat. Neurosci. 9, 999-1000. https://doi.org/ 10.1038/nn1735.

Gorczyca, D.A., Younger, S., Meltzer, S., Kim, S.E., Cheng, L., Song, W., Lee, H.Y., Jan, L.Y., Jan, Y.N., 2014. Identification of Ppk26, a DEG/ENaC channel functioning with Ppk1 in a mutually dependent manner to guide locomotion behavior in drosophila. Cell Rep. 9, 1446-1458. https://doi.org/10.1016/j.celrep.2014.10.034.

Grabherr, M.G., Haas, B.J., Yassour, M., Levin, J.Z., Thompson, D.A., Amit, I., Adiconis, X., Fan, L., Raychowdhury, R., Zeng, Q., Chen, Z., Mauceli, E., Hacohen, N., Gnirke, A., Rhind, N., di Palma, F., Birren, B.W., Nusbaum, C., Lindblad-Toh, K., Friedman, N., Regev, A., 2011. Full-length transcriptome assembly from RNA-Seq data without a reference genome. Nat. Biotechnol. 29, 644-652. https://doi.org/10.1038/nbt.1883.

Green, J.E., Cavey, M., Médina Caturegli, E., Aigouy, B., Gompel, N., Prud'homme, B., 2019. Evolution of Ovipositor Length in Drosophila suzukii Is Driven by Enhanced Cell Size Expansion and Anisotropic Tissue Reorganization. Curr. Biol. 29, 20752082.e6. https://doi.org/10.1016/j.cub.2019.05.020.

Guo, Y., Wang, Y., Wang, Q., Wang, Z., 2014. The role of PPK26 in drosophila larval mechanical nociception. Cell Rep. 9, 1183-1190. https://doi.org/10.1016/j.celrep. 2014.10.020.

Guo, Y., Wang, Y., Zhang, W., Meltzer, S., Zanini, D., Yu, Y., Li, J., Cheng, T., Guo, Z., Wang, Q., Jacobs, J.S., Sharma, Y., Eberl, D.F., Göpfert, M.C., Jan, L.Y., Jan, Y.N., Wang, Z., 2016. Transmembrane channel-like (tmc) gene regulates Drosophila larval locomotion. Proc. Natl. Acad. Sci. U.S.A. 113, 7243-7248. https://doi.org/10.1073/ pnas. 1606537113.

Hall, T., 1999. BioEdit: a user-friendly biological sequence alignment editor and analysis program for Windows 95/98/NT. Nucleic Acids Symp. Ser. 41, 95-98 https:// doi.org/citeulike-article-id:691774.

Hauser, M., 2011. A historic account of the invasion of Drosophila suzukii (Matsumura) (Diptera: Drosophilidae) in the continental United States, with remarks on their identification. Pest Manag. Sci. 67, 1352-1357. https://doi.org/10.1002/ps.2265.

Hickner, P.V., Rivaldi, C.L., Johnson, C.M., Siddappaji, M., Raster, G.J., Syed, Z., 2016. The making of a pest: Insights from the evolution of chemosensory receptor families in a pestiferous and invasive fly, Drosophila suzukii. BMC Genomics 17, 1-17. https://doi.org/10.1186/s12864-016-2983-9.

Hu, Y., Wang, Z., Liu, T., Zhang, W., 2019. Piezo-like gene regulates locomotion in drosophila larvae. Cell Rep. 26, 1369-1377.e4. https://doi.org/10.1016/j.celrep.2019. 01.055.

Ioriatti, C., Walton, V., Dalton, D., Anfora, G., Grassi, A., Maistri, S., Mazzoni, V., 2015. Drosophila suzukii (Diptera: Drosophilidae) and Its Potential Impact to wine grapes during harvest in two cool climate wine grape production regions. J. Econ. Entomol. 108, 1148-1155. https://doi.org/10.1093/jee/tov042.

Jang, W., Lee, S., Choi, S.I., Chae, H.S., Han, J., Jo, H., Hwang, S.W., Park, C.S., Kim, C., 2019. Impairment of proprioceptive movement and mechanical nociception in Drosophila melanogaster larvae lacking Ppk30, a Drosophila member of the Degenerin/Epithelial Sodium Channel family. Genes Brain Behav. 18, 1-8. https:// doi.org/10.1111/gbb.12545.

Johnson, B.R., Atallah, J., Plachetzki, D.C., 2013. The importance of tissue specificity for RNA-seq: Highlighting the errors of composite structure extractions. BMC Genomics 14, 1. https://doi.org/10.1186/1471-2164-14-586.

Joseph, R.M., Carlson, J.R., 2015. Drosophila chemoreceptors: a molecular interface between the chemical world and the brain. Trends Genet. 31, 683-695. https://doi. org/10.1016/j.tig.2015.09.005.

Kaessmann, H., 2010. Origins, evolution, and phenotypic impact of new genes. Genome Res. 20, 1313-1326. https://doi.org/10.1101/gr.101386.109.

Karageorgi, M., Bräcker, L.B., Lebreton, S., Minervino, C., Cavey, M., Siju, K.P., Grunwald Kadow, I.C., Gompel, N., Prud'homme, B., 2017. Evolution of Multiple Sensory Systems Drives Novel Egg-Laying Behavior in the Fruit Pest Drosophila suzukii. Curr. Biol. 27, 847-853. https://doi.org/10.1016/j.cub.2017.01.055.

Karkali, K., Martin-Blanco, E., 2017. Mechanosensing in the Drosophila nervous system. Semin. Cell Dev. Biol. 71, 22-29. https://doi.org/10.1016/j.semcdb.2017.06.014.

Keil, T.A., Steinbrecht, R.A., 1984. Mechanosensitive and Olfactory Sensilla of Insects, in: Insect Ultrastructure. Springer US, pp. 477-516. https://doi.org/10.1007/978-14613-2715-8 13.

Kernan, M.J., 2007. Mechanotransduction and auditory transduction in Drosophila. Pflugers Arch. Eur. J. Physiol. 454, 703-720. https://doi.org/10.1007/s00424-0070263-x.

Kim, S.E., Coste, B., Chadha, A., Cook, B., Patapoutian, A., 2012. The role of drosophila piezo in mechanical nociception. Nature 483, 209-212. https://doi.org/10.1038/ nature10801.

Kinjo, H., Kunimi, Y., Ban, T., Nakai, M., 2013. Oviposition efficacy of drosophila suzukii (diptera: drosophilidae) on different cultivars of blueberry. J. Econ. Entomol. 106, 1767-1771. https://doi.org/10.1603/ec12505.

Langmead, B., Salzberg, S.L., 2012. Fast gapped-read alignment with Bowtie 2. Nat. Methods 9, 357-359. https://doi.org/10.1038/nmeth.1923.

Lee, H., Choi, H.W., Zhang, C., Park, Z.Y., Kim, Y.J., 2016. A pair of oviduct-born Pickpocket neurons important for egg-laying in Drosophila melanogaster. Mol. Cells 39, 573-579 https://doi.org/10.14348/molcells.2016.0121.

Lee, J.C., Bruck, D.J., Curry, H., Edwards, D., Haviland, D.R., Van Steenwyk, R.A., Yorgey, B.M., 2011. The susceptibility of small fruits and cherries to the spotted-wing drosophila, Drosophila suzukii. Pest Manag. Sci. 67, 1358-1367. https://doi.org/10. 1002/ps.2225.

Li, B., Dewey, C.N., 2011. RSEM: accurate transcript quantification from RNA-Seq data with or without a reference genome. BMC Bioinf. 12, 323. https://doi.org/10.1186/ 1471-2105-12-323.

Marshall, K.L., Lumpkin, E.A., 2012. The molecular basis of mechanosensory transduction, in: Lopez-Larrea, C. (Ed.), Sensing in Nature. pp. 142-155.

Mi, H., Huang, X., Muruganujan, A., Tang, H., Mills, C., Kang, D., Thomas, P.D., 2017. PANTHER version 11: expanded annotation data from Gene Ontology and Reactome pathways, and data analysis tool enhancements. Nucleic Acids Res. 45, D183-D189. https://doi.org/10.1093/nar/gkw1138.

Muto, L., Kamimura, Y., Tanaka, K.M., Takahashi, A., 2018. An innovative ovipositor for niche exploitation impacts genital coevolution between sexes in a fruit-damaging Drosophila. Proc. R. Soc. B Biol. Sci. 285, 20181635. https://doi.org/10.1098/rspb. 2018.1635.

Naccarati, C., Audsley, N., Keen, J.N., Kim, J.H., Howell, G.J., Kim, Y.J., Isaac, R.E., 2012 The host-seeking inhibitory peptide, Aea-HP-1, is made in the male accessory gland and transferred to the female during copulation. Peptides $34,150-157$. https://doi. org/10.1016/j.peptides.2011.10.027.

Oliveros, J.C., 2015. Venny. An interactive tool for comparing lists with Venn's diagrams [WWW Document]. URL http://bioinfogp.cnb.csic.es/tools/venny/index.html.

Ramasamy, S., Ometto, L., Crava, C.M., Revadi, S., Kaur, R., Horner, D., Pisani, D., Dekker, T., Anfora, G., Rota-Stabelli, O., 2016. The evolution of olfactory gene families in Drosophila and the genomic basis of chemical-ecological adaptation in Drosophila suzukii. Genome Biol. Evol. 8, 2297-2311. https://doi.org/10.1093/gbe/ evw160.

Sato, K., Tanaka, K., Touhara, K., 2011. Sugar-regulated cation channel formed by an insect gustatory receptor. Proc. Natl. Acad. Sci. U.S.A. 108, 11680-11685. https:// doi.org/10.1073/pnas.1019622108.

Schindelin, J., Arganda-Carreras, I., Frise, E., Kaynig, V., Longair, M., Pietzsch, T., Preibisch, S., Rueden, C., Saalfeld, S., Schmid, B., Tinevez, J.-Y., White, D.J., Hartenstein, V., Eliceiri, K., Tomancak, P., Cardona, A., 2012. Fiji: an open-source platform for biological-image analysis. Nat. Methods 9, 676-682. https://doi.org/10. 
1038/nmeth.2019.

Shearer, P.W., West, J.D., Walton, V.M., Brown, P.H., Svetec, N., Chiu, J.C., 2016. Seasonal cues induce phenotypic plasticity of Drosophila suzukii to enhance winter survival. BMC Ecol. https://doi.org/10.1186/s12898-016-0070-3.

Stocker, R.F., 1994. The organization of the chemosensory system in Drosophila melanogaster: a rewiew. Cell Tissue Res. 275, 3-26. https://doi.org/10.1007/ BF00305372.

Tabarean, I.V., Morris, C.E., 2002. Membrane stretch accelerates activation and slow inactivation in Shaker channels with S3-S4 linker deletions. Biophys. J . 82, 2982-2994. https://doi.org/10.1016/S0006-3495(02)75639-7.

Taylor, B.J., 1989a. Sexually dimorphic neurons of the terminalia of drosophila melanogaster: II. Sex-specific axonal arborizations in the central nervous system. J. Neurogenet. 5, 193-213. https://doi.org/10.3109/01677068909066208.

Taylor, B.J., 1989b. Sexually dimorphic neurons in the terminalia of drosophila melanogaster: I. Development of sensory neurons in the genital disc during metamorphosis. J. Neurogenet. 5, 173-192. https://doi.org/10.3109/ 01677068909066207.

Tracey, W.D., Wilson, R.I., Laurent, G., Benzer, S., 2003. Painless, a Drosophila Gene Essential for Nociception. Cell 113, 261-273.

Tsubouchi, A., Caldwell, J.C., Tracey, W.D., 2012. Dendritic filopodia, ripped pocket, NOMPC, and NMDARs contribute to the sense of touch in Drosophila larvae. Curr. Biol. 22, 2124-2134. https://doi.org/10.1016/j.cub.2012.09.019.

Tuthill, J.C., Wilson, R.I., 2016. Mechanosensation and adaptive motor control in insects. Curr. Biol. 26, R1022-R1038, https://doi.org/10.1016/j.cub.2016.06.070.

Walker, R.G., Willingham, A.T., Zuker, C.S., 2000. Drosophila Mechanosensory Transduction Channel. Science (80-.). 287, 2229-2234.

Zhong, L., Hwang, R.Y., Tracey, W.D., 2010. Pickpocket Is a DEG/ENaC Protein Required for Mechanical Nociception in Drosophila Larvae. Curr. Biol. 20, 429-434. https:// doi.org/10.1016/j.cub.2009.12.057. 